\title{
Fluorescence light detection with the FAMOUS telescope
}

\author{
Adrianna García ${ }^{* 1}$, Thomas Bretz ${ }^{1}$, Paulo Ferreira ${ }^{1}$, Thomas Hebbeker ${ }^{1}$, Julian \\ Kemp ${ }^{1}$, Tobias Pan ${ }^{1}$, Christine Peters ${ }^{1}$, Merlin Schaufel ${ }^{1}$, Johannes Schumacher ${ }^{1}$ \\ ${ }^{1}$ RWTH Aachen University, Sommerfeldstraße, D-52074 Aachen \\ E-mail: garcia@physik.rwth-aachen.de
}

\begin{abstract}
The First Auger Multi-pixel photon counter camera for the Observation of Ultra-high-energy airShowers (FAMOUS) is a telescope developed at the RWTH Aachen University in Germany. Its main goal is to measure photons produced in extensive air showers generated from the interaction of ultra high energy cosmic rays with the Earth's atmosphere. FAMOUS uses Silicon Photomultipliers as photodetection device instead of Photomultiplier tubes used by the fluorescence telescope in the Pierre Auger Observatory. The data acquisition system currently used by FAMOUS is suitable to measure Cherenkov photons. Now we evaluate its capability to detect also fluorescence light which will give information about the energy and the arrival direction of the primary cosmic rays.
\end{abstract}

36th International Cosmic Ray Conference -ICRC2019-

July 24th - August 1st, 2019

Madison, WI, U.S.A.

\footnotetext{
* Speaker.
} 


\section{Introduction}

Cosmic rays (CRs) are energetic particles that are bombarding the Earth constantly. Their detection can be done directly by using satellites but, due to their low flux, it is limited by the size of the satellites. In order to compensate the low fluxes at high energies, it is necessary to increase the detection area. Indirect detection from the ground is the only possibility to achieve the required areas.

When a cosmic ray penetrates the Earth's atmosphere a cascade of secondary particles is produce. This so-called extensive air shower (EAS) is composed mainly of charged particles and (fluorescence and Cherenkov) photons travelling through the atmosphere at relativistic speed. The secondary particles, mostly electrons and positrons, excite the molecules in the atmosphere during their path. In the de-excitation process, fluorescence photons will be emitted isotropically in the wavelength range between $300 \mathrm{~nm}$ and $430 \mathrm{~nm}$, corresponding to the emission lines of Nitrogen which approximately accounts for $80 \%$ of the composition of the Earth's atmosphere.

Fluorescence telescopes collect the emitted photons to image the air showers. The number of photons collected by fluorescence telescopes is a calorimetric measurement since the number of emitted photons is proportional to the number of particles in the shower, at the same time the number of particles is proportional to the energy of the primary cosmic ray. Therefore, the number of photons, $N_{\gamma}$, generated in an EAS can be expressed by:

$$
\frac{d^{2} N_{\gamma}}{d X d \lambda}=Y(\lambda, P, T, u) \times \frac{d E_{d e p}^{t o t}}{d X}
$$

being $Y$ the fluorescence yield that depends on the wavelength $(\lambda)$, pressure $(P)$, the temperature $(T)$ and the humidity $(u)$ of the atmosphere, and $E_{\text {dep }}^{\text {tot }}$ the total energy deposited in the atmosphere.

The Pierre Auger Observatory (PAO), located in Malargüe - Argentina, is the largest observatory dedicated to study cosmic rays. It comprises 1660 water Cherenkov detector stations distributed in an area of $3000 \mathrm{~km}^{2}$, enabling to detect the most energetic cosmic rays, so-called ultra high energy cosmic rays (UHECRs), covering an energy range from $10^{17} \mathrm{eV}$ to $10^{20} \mathrm{eV}$ [1]. With its 27 fluorescence telescopes overseeing the whole array, it is possible to record information about CRs by using the fluorescence technique. Each telescope is equipped with a camera of 440 photomulplier tubes (PMTs) and an array of mirrors that focuses the light into the camera. Nevertheless, the use of PMTs carries a number of considerable drawbacks. They have to be operated under high voltage $(\sim 1000 \mathrm{~V})$, are affected by magnetic fields, have limited photodetection efficiency, and they are fragile devices that can be severely damage when exposed to high light fluxes. This limits the maximum brightness at which they can be operated without risk which reduce significantly the duty cycle of the fluorescence telescopes. In recent years, alternatives to replace PMTs have become mature and are considered when developing new detectors [2]. In this context, Silicon Photomultipliers (SiPMs) appear as the most promising candidates to replace PMTs, more in [3].

A Silicon Photomultiplier (SiPM) is a solid-state photodiode that consists of small-sized microcells connected in parallel inside a silicon substrate [4]. Some of the remarkable features of this detector is the ability to detect single photons in a very short time window which make them suitable for the detection of photons produced in the EAS. Additionally, they have low costs in 
massive production. SiPMs are produced in small sizes, typically few $\mathrm{mm}^{2}$. To increase their effective photodetection area, light-guides can be used to focus the light from a larger surface onto the sensitive area.

In particular, SiPMs have been tested in the First G-APD Cherenkov Telescope (FACT) operated on the Canary Island of La Palma [3]. With the invention of new optical telescopes with cameras manufactured with SiPMs, the possibility to create low-cost telescopes in a massive production increased. One example is the the First Auger Multi-pixel photon counter camera for the Observation of Ultra-high-energy air-Showers (FAMOUS) designed and built by the III. Physikalisches Institut A, RWTH Aachen University in Germany [5, 6, 7].

\section{FAMOUS}

FAMOUS is a refracting telescope developed to detect the fluorescence light generated in air showers produced by UHECRs. Its main structure is a tube made of carbon fiber reinforced plastic that holds a Fresnel lens of $549.7 \mathrm{~mm}$ of diameter with a focal distance of $502.1 \mathrm{~mm}$ where a camera composed of a hexagonal array of 64 SiPMs is placed, see Figure 1. The SiPMs used by the telescope are Hamamatsu S10985-100C. Each of them consists of an array of four SiPMs with $3 \times 3 \mathrm{~mm}^{2}$ area and a size cell of $100 \mu \mathrm{m}$. In [8] more details of the telescope are given.

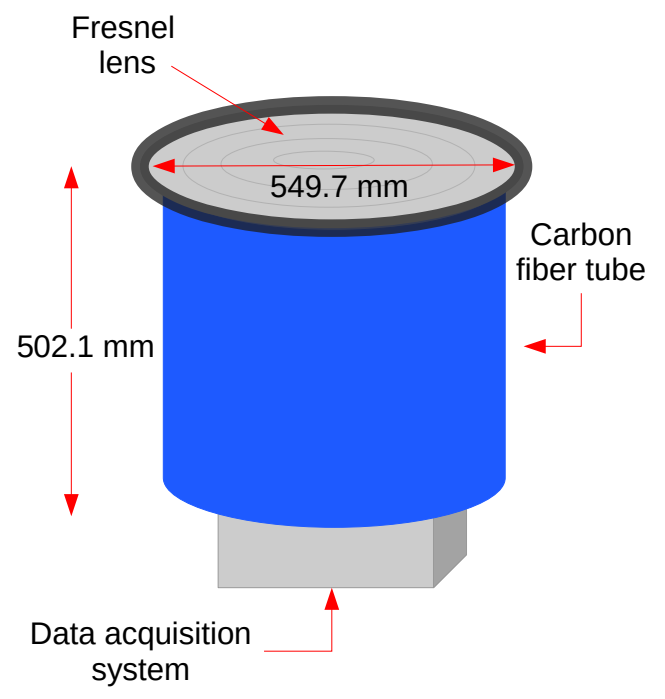

Figure 1: Sketch of the FAMOUS telescope.

In order to increase the detection area of the SiPMs, on top of each sensor a hollow Winston cone made of Aluminum has been placed [5, 8]. It is a light collector whose design consists of a parabolic cone with a circular entrance of $6.7 \mathrm{~mm}$ radius, a circular exit of $3 \mathrm{~mm}$ radius and a lenght of $19.6 \mathrm{~mm}$, providing a field of view (FOV) of $1.5^{\circ}$ each. In total, 64 SiPMs are combined giving a total FOV of $12^{\circ}$, see Figure 2 . 




Figure 2: Sketch of the telescope camera array with the Winston cones, taken from [9].

\subsection{Data acquisition}

The properties of the tracks produced in the camera by Cherenkov and fluorescence light are quite different. Specifically, the time window at which each phenomenon occurs, differs by orders of magnitude. This is a crucial characteristic when selecting a data acquisition system (DAQ).

The current DAQ used by FAMOUS has been inherited from the FACT telescope which measures faint light flashes lasting few nanoseconds (Cherenkov light). In this DAQ, the detected signal is sampled by a Domino Ring Sampling chip, DRS4 with a sampling frequency of $2 \mathrm{GHz}$ taking 1024 samples per trigger. Thus, a total of $512 \mathrm{~ns}$ is sampled.

The capability of fluorescence detection with the FACT DAQ can be checked with a geometrical study of the arrival time of photons coming from an EAS. Figure 3 shows a sketch of the geometrical assumptions that are made in this study. Simulations have been done varying the shower incident angles and the height at which it crosses the telescopes axis. Figure 4a shows the time interval of the trace produced by a shower in the FOV of the telescope as a function of the angle $\beta$ for different heights. Assuming isotropically orientated shower axes, Figure $4 \mathrm{~b}$ shows the distribution of the time intervals. Typically, events are expected to last a few of $\mu$ s up tp more than $10 \mu$ s in the FOV, i.e., three orders of magnitude above the time window which the current DAQ supports. This motivates the development of a new DAQ which covers the sampling rate required to measure fluorescence light.

The event rate for FAMOUS can be estimated by comparison with the event rate of other well characterized telescopes. Knowing the solid angle $(\Omega)$, the energy threshold $\left(E_{t h}\right)$ of both telescopes and the event rate of the additional telescope $(R)$, the fluorescence rate will be given by:

$$
\frac{R_{1}}{R_{2}} \propto\left(\frac{E_{t h, 1}}{E_{t h, 2}}\right)^{-2} \frac{\Omega_{1}}{\Omega_{2}}
$$




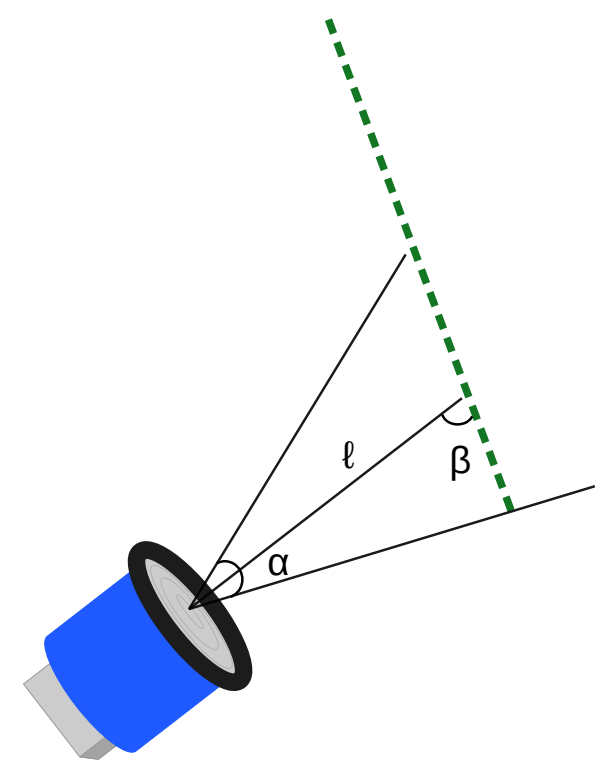

Figure 3: Sketch of the geometrical parameters considered for the study of the fluorescence event time in the FOV of FAMOUS. $\alpha$ is the FOV of the telescope, $\beta$ is the inclination angle of the shower axis with respect to the telescope axis and $\ell$ is the distance from the telescope to the shower along the telescope axis, i. e., the height at which the EAS crosses the FOV of the telescope.

with the exponent, -2 , being the integral spectral index for cosmic rays with energies above $10^{18} \mathrm{eV}$.

For FAMOUS, the experimental energy threshold is not set yet. An easy way to estimate it is considering its proportionality with respect to the optical signal to noise ratio. It is expressed as $S N R \propto\left(E_{t h}\right)^{-1}$, with $S N R \propto \sqrt{\varepsilon A / d \Omega}$ where $\varepsilon$ and $A$ are the optical efficiency and the effective detection area of the telescope, respectively. Finally, $d \Omega$ is the solid angle of each camera pixel. Therefore, the equation 2.1 can be written as:

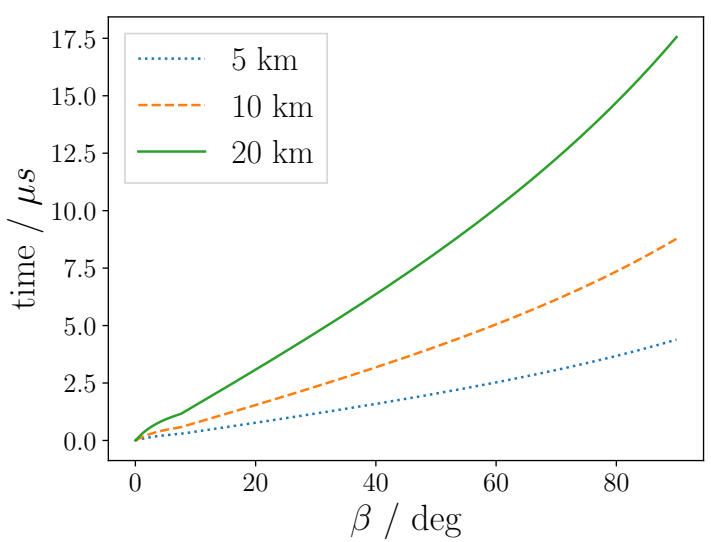

(a)

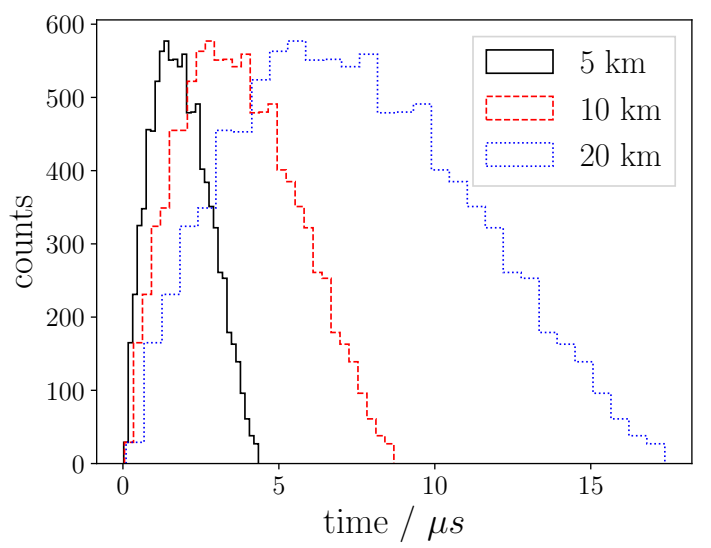

(b)

Figure 4: (a) Time interval of a typical fluorescence event in the FOV of FAMOUS as a function of the angle $\beta$ for different heights, see Figure 3. (b) Distribution of the time intervals for the different heights considered. 


$$
\frac{R_{1}}{R_{2}} \propto \frac{\varepsilon_{1}}{\varepsilon_{2}} \cdot \frac{A_{1}}{A_{2}} \cdot \frac{d \Omega_{2}}{d \Omega_{1}} \cdot \frac{\Omega_{1}}{\Omega_{2}}
$$

The fluorescence telescopes of the Pierre Auger Observatory are used as reference. Every telescope has a solid angle of $0.21 \mathrm{sr}$, an aperture of $1.5 \mathrm{~m}^{2}$ and an event rate of $2 \mathrm{mHz}$ above the energy threshold $\left(10^{18} \mathrm{eV}\right.$ [1]). Assuming that the optical efficiency of each telescope is the same and taking into account that the pixel solid angles are equal, using equation 2.2 a rate of 0.19 events per hour is obtained for the FAMOUS telescope. If we consider an observation time of around $8 \mathrm{~h}$ per night, the total number of CR events per night detected by FAMOUS would be approximately 2 . This value represents a lower limit for FAMOUS and it can be improved by increasing the observation time during nights with moon light, which can be achieved with the SiPM camera.

\section{Monte Carlo simulations of the expected event rate}

Monte Carlo simulations with CONEX [10] have been done to obtain the event rate for FAMOUS. A total of 1164506 proton, helium and iron primary particles have been simulated in an energy range between $10^{17} \mathrm{eV}$ and $10^{21} \mathrm{eV}$ with a spectral index equal to -1 using EPOSLHC and FLUKA as high and low energy hadronic interaction models, respectively. The zenith and azimuth angle distribution have been chosen between $0^{\circ}$ and $80^{\circ}$, and $0^{\circ}$ and $360^{\circ}$, respectively. Afterwards, the energy spectrum of the simulated CRs has been parametrized as a power-law with smooth change of the spectral index which was fitted to the data of the Pierre Auger Observatory [11].

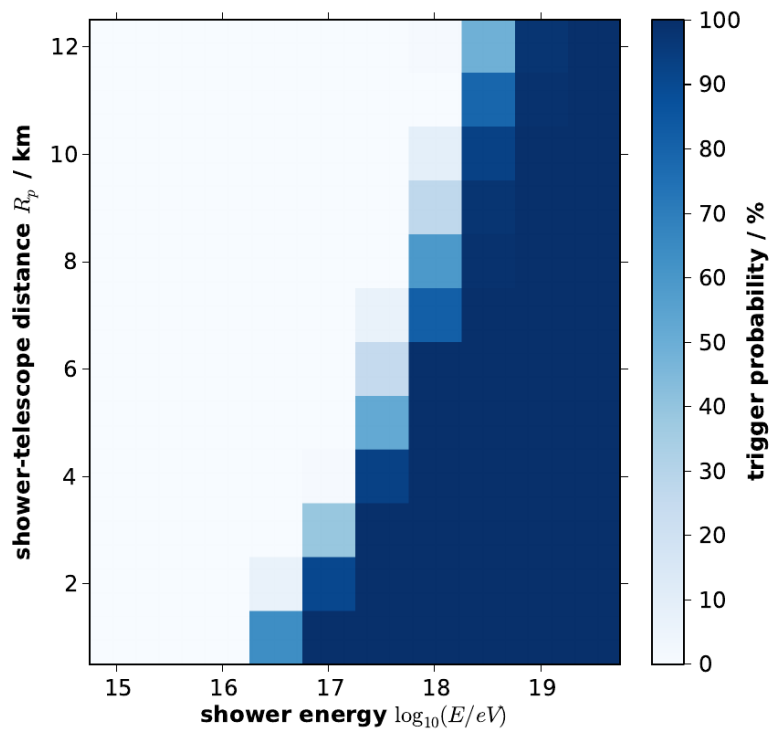

Figure 5: Simulated trigger probability for vertical shower detected by FAMOUS as a function of the air shower energy and the shower core to telescope distance, taken from [5].

In order to estimate the best angle of inclination of the telescope to have a high efficiency in the detection of EAS, the shower core position at ground is randomized. The event rate is estimated 
by counting the number of events where the shower maximum lies in the FOV. Additionally, the trigger detection efficiency is taken into account considering the number of detected fluorescence photons as shown in Figure 5 [5]. The resulting expected number of EAS detected per hour as a function of the telescope tilting angle for different primary CR energies is shown in Figure 6. For an energy threshold of $10^{17} \mathrm{eV}$, the most favored tilt angle of the telescope lies between $0^{\circ}$ and $30^{\circ}$ to maximize the total number of detected air shower events per hour. In this setup, FAMOUS is capable to detect almost two events per hour. At higher energies, the showers are penetrating deep in the atmosphere, as a consequence a bigger tilt angle of the telescope is preferred to maximize the event rate at these energies.

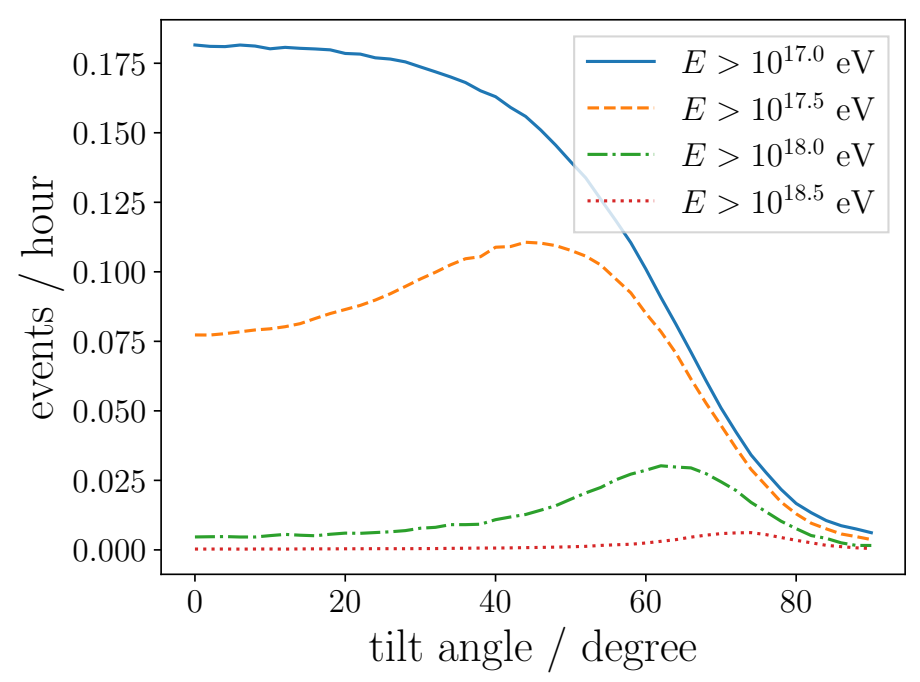

Figure 6: Expected air shower events per hour detected by FAMOUS as a function of the tilt angle for four different energy thresholds.

\section{Summary and outlook}

In order to explore the fluorescence detection capabilities of the telescope with the current DAQ, an effective test is represented by pointing a UV laser into the sky. The laser beam will be scattered off the particles in the atmosphere allowing to mimic the isotropic emission of fluorescence photons. For the test, an UV laser of class IV with a wavelength of $337.1 \mathrm{~nm}$ and a peak power of $500 \mathrm{~kW}$ at $2 \mathrm{~ns}$ pulse length is considered. Lasers with these features have several applications, the most outstanding being the monitoring of the atmosphere and 3D mapping of the Earth ("LIDAR") [12]. Being a class IV laser, a special permission is needed to operate it, the request of such a permission is still in progress.

The possibility to exchange the current DAQ has been discussed, resulting in the EASIROC (Extended Analogue Silicon-pm Integrated ReadOut Chip) [13] as a suitable option for the implementation of the readout system for FAMOUS. The EASIROC is an analogue front end with 32 input channels designed specifically for the readout of SiPM signals in experiments like PEBS and MuRAY [13]. 
In addition, a test at the Pierre Auger Observatory has been proposed in order to calibrate FAMOUS and to establish its trigger threshold for fluorescence light by measuring in coincidence with its well understood fluorescence telescopes.

In order to progress towards the measurements in the field, dedicated simulations of the telescope are planed to be developed by using the already implemented Geant 4 simulations [5, 14] and the Offline Software Framework of the Pierre Auger Observatory [15]. In this framework, it is possible to simulate EAS produced in the observatory environment and reconstruct the shower parameters in order to study the shower and therefore, the primary cosmic ray properties.

The FAMOUS telescope has been presented as a design for the future generation of fluorescence telescopes by using cameras built with SiPMs. This innovative design promises to increase their duty cycle, as well as to manufacture cheap and easy to reproduce telescopes, bringing the opportunity to develop fluorescence telescopes arrays that can oversee huge areas for a low monetary investment.

Simulations and experimental setups are being prepared in order to test FAMOUS at the Pierre Auger Observatory. Preliminary results have been shown to motivate the replacement of the current DAQ of FAMOUS, which will allow to record the first fluorescence light measured with FAMOUS at this observatory.

\section{References}

[1] J. Abraham et al., Nucl. Instrum. Methods Phys. Res. A 620 (2010) 227-251, [DOI:0.1016/j.nima.2010.04.023].

[2] T. C. Weekes, CRC Press (2003), [DOI:10.1201/9781420033199].

[3] A. Biland et al., JINST 9 (2014) P10012, [DOI:10.1088/1748-0221/9/10/P10012].

[4] P. Eckert et al., Nucl. Instrum. Methods Phys. Res. A, 620 (2010) 217-226, [DOI:10.1016/j.nima.2010.03.169].

[5] T. Niggemann et al., In Proc. of the 33th ICRC 2013, [arXiv:1502.00792].

[6] T. Bretz et at., PoS(ICRC2015) 649, [DOI:10.22323/1.236.0649].

[7] J. Kemp et al., PoS(ICRC2017) 466, [DOI:10.22323/1.301.0466].

[8] T. Bretz et al., JINST 13 (2018) P07024, [DOI:10.1088/1748-0221/13/07/P07024].

[9] T. Niggemann, PhD Thesis, RWTH Aachen University (2017).

[10] D. Heck et al., Report FZKA 6019 (1998).

[11] F. Fenu [Pierre Auger Collaboration], PoS(ICRC2017) 486, [DOI:10.22323/1.301.0486]

[12] R. T. H. Collis, Science, Volume 149, Issue 3687 (1995) 978-981, [DOI:10.1126/science.149.3687.978].

[13] S. Callier et al., Physics Procedia 37 (2012) 1569-1576, [DOI:10.1016/j.phpro.2012.02.486].

[14] S.Agostinelli et al., Nucl. Instrum. Methods Phys. Res. A 506 (2003) 250-303, [DOI:10.1016/S0168-9002(03)01368-8].

[15] S. Argirò et al., Nucl. Instrum. Methods Phys. Res. A 580 (2007) 1485-1496, [DOI:10.1016/j.nima.2007.07.010]. 